\title{
Adaptation des littoraux à l'élévation prévisible du niveau des mers : Spécificités climatiques, humaines, environnementales et d'urbanisme réglementaire pour les outre-mer
}

\author{
Jean-Marc BEYNET ${ }^{1}$, Charles EGRETAUD ${ }^{2}$, Jean-Pierre REDON ${ }^{3}$
}

1. Beynet-Consult, 35 rue de Peyrouse, 30320 Marguerittes, France. beynet.consult@gmail.com

2. Société Pae Tai - Pae Uta, Rue Vénus, BP1746, 98713 Papeete, Polynésie française. charles.egretaud@ptpu.pf

3. Legal Consultant \& Partners, Plaza Universitat 3, Barcelona, 08007, Espagne.

legalconsultant.partners@gmail.com

\section{Résumé :}

Au niveau national, le SHOM gère de nombreux marégraphes, y compris outre-mer. Sur le site refmar.shom.fr, la hausse du niveau de la mer (issue du rapport spécial du GIEC sur l'océan et la cryosphère dans le contexte du changement climatique, OPPENHEIMER et al., 2019) est précisée : d'ici 2100, les scientifiques estiment désormais que le niveau marin pourrait augmenter de 29 à $110 \mathrm{~cm}$ suivant les modèles. Et malgré ces risques de submersion marine, les populations ne cessent de se rapprocher des bandes littorales. C'est considérable pour le littoral français métropolitain. Mais qu'en sera-t-il dans les outre-mer? Pour tenter d'y répondre le présent article passe en revue et souligne les spécificités des outre-mer par rapport aux littoraux métropolitains aussi bien pour le volet climatique (risques cycloniques), le volet humain (les populations riveraines connaissent déjà les problèmes de submersion marine en raison des marées de tempête), le volet environnemental (lagons et présence de barrières récifales dans certains cas, mangroves dans d'autres) et enfin le volet de l'urbanisme réglementaire. L'article aborde en particulier le cas des Antilles françaises et de la Polynésie française.

Mots-clés :

Elévation du niveau des mers, Habitat flottant ou sur pilotis, GIZC, Hydrodynamique, Environnement littoral, Lagons et barrières de récifs, Contexte juridico-administratif, Urbanisme réglementaire

\section{Impact du réchauffement climatique dans les outremers : Prise en compte des aspects humains, hydrodynamique et environnement}

Dans le Pacifique, les atolls, ont déjà été totalement submergés lors des périodes de réchauffement entre les périodes glaciaires. En raison de l'alternance des cycles de Milankovitch, le niveau de l'océan aurait varié de près de $130 \mathrm{~m}$, descendant de $90 \mathrm{~m}$ environ et remontant de plus de $40 \mathrm{~m}$ parfois, submergeant les atolls. Les atolls sont en effet des îles coralliennes qui dépassent à peine le niveau de l'océan et dont la formation 


\section{Thème 7 - Risques côtiers}

peut être expliquée par la subsidence du plancher océanique du fait de son refroidissement par diffusion (LABROSSE, 2010). On en retrouve des traces, par exemple dans l'atoll de Makatea, avec les différents rivages fossiles au sud ou les méga blocs (féo) sur le récif de certains atolls, toujours au sud-ouest. Ces variations sont à l'origine de nombreuses lignes de rivages fossiles différentes, que l'on retrouve également sur Tahiti, par exemple ou de spéciations d'oiseaux aux Tuamotu, en fonction du retour à l'exondation.

\subsection{En Polynésie}

L'habitat pré européen était situé essentiellement en vallée, pour des raisons de défense et d'étroitesse de la bande littorale. Le développement littoral de l'habitat est venu avec les européens, s'articulant le long de la route de ceinture, souvent gagnée sur le lagon dans les îles hautes. Avec le tourisme et la pression foncière, du fait d'un marnage et un risque cyclonique faibles, des constructions se sont développées sur l'eau, à Bora Bora, lors du tournage du Bounty, avec un complexe devenu un hôtel toujours en place et l'installation de bungalows sur pilotis dans la partie sud, le tout sous impulsions d'américains. Le concept s'est étendu sur l'île de Bora Bora, avant de s'exporter par la suite sur Moorea, Tahaa ou Raiatea, mais très peu sur Tahiti ( 6 unités à Punanuia, figure 1). Toujours en Polynésie, le lien à la terre, matérialisé par l'enfouissement du placenta et la plantation d'un arbre lors de l'arrivée d'un nouveau-né, a plus conduit les polynésiens à la pratique du remblai sur le lagon, avec un impact environnemental désastreux en termes de nurseries piscicoles. Cette pratique est depuis une dizaine d'années fortement encadrée et très limitée, bien que dans certains cas, elle apparait incontournable pour réduire les indentations génératrices de confinement des eaux.
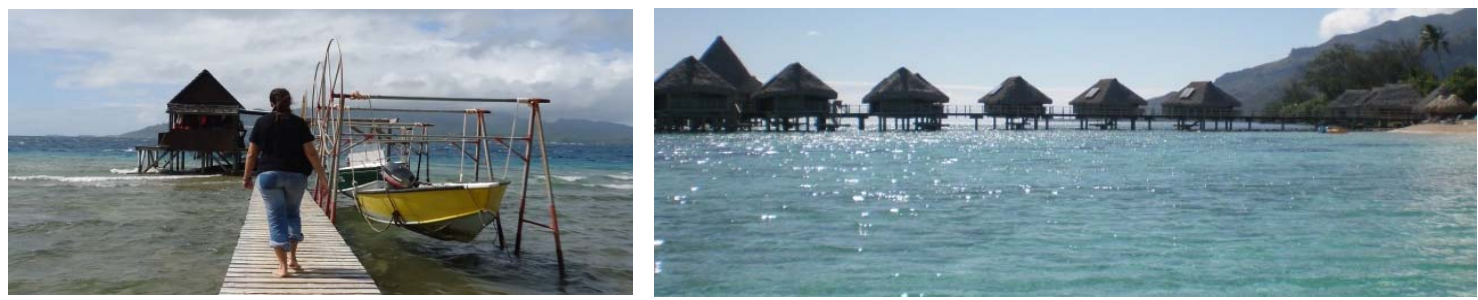

Figure 1. Atelier artisanal sur pilotis et bateaux hors d'eau à Tahaa - Hôtel sur pilotis à Bora-Bora, (clichés ${ }^{\circledR}$ J.-M. Beynet, 2012).

Étrangement, les constructions sur pilotis ont une mauvaise image en Polynésie. Le remblai dont l'incidence environnementale est démontrée conserve une bonne réputation. Les techniques innovatrices conduisant à des développements d'unités sur deux étages, avec piscine et spa sur pilotis restent l'apanage des constructions hôtelières. Le savoirfaire technique mais également des entreprises polynésiennes reste confiné sur la Polynésie. 


\section{XVİ̀mes Journées Nationales Génie Côtier - Génie Civil \\ Le Havre 2020}

\subsection{Aux Antilles}

La culture du risque par les élus et les populations a déjà fait l'objet d'études et de publications (PAGNEY BENITO-ESPINAL, 2019). Cette "culture du risque au sens strict est la connaissance qui permet aux acteurs d'adopter des comportements adaptés en cas de catastrophe. Dans les Antilles françaises, des acteurs divers, publics notamment, contribuent par la prévention à forger cette culture au sein de la population. Pourtant, si les risques sont présents et visibles dans le paysage et dans le quotidien, un effet d'accoutumance tend à favoriser des comportements individuels de déni". Dans les Antilles, (figure 2), la population qui vit proche de la mer a déjà l'habitude des submersions marines suite au passage des cyclones qui, en plus des fortes houles au large déferlantes en arrivant sur les littoraux. Ces houles cycloniques sont concomitantes avec une dépression barométrique qui peut engendrer 1 à $2 \mathrm{~m}$ de surélévation de la mer, (marée de tempête), voire plus parfois, surtout dans les zones plus ou moins confinées comme les culs-de-sac marins en Guadeloupe par exemple.

Aussi, la surélévation prévisible lente (quelques millimètres par an ou 0,60 à $1,10 \mathrm{~m}$ en 80 ans comme le prédit le GIEC (OPPENHEIMER et al., 2019) est presque négligeable eu égard aux marées de tempête brutales lors du passage d'une dépression cyclonique. Les populations sont peut-être plus aptes à y faire face que les métropolitains dans des zones littorales très basses comme une partie de la Normandie, la Charente-Maritime, l'Occitanie et surtout La Camargue.

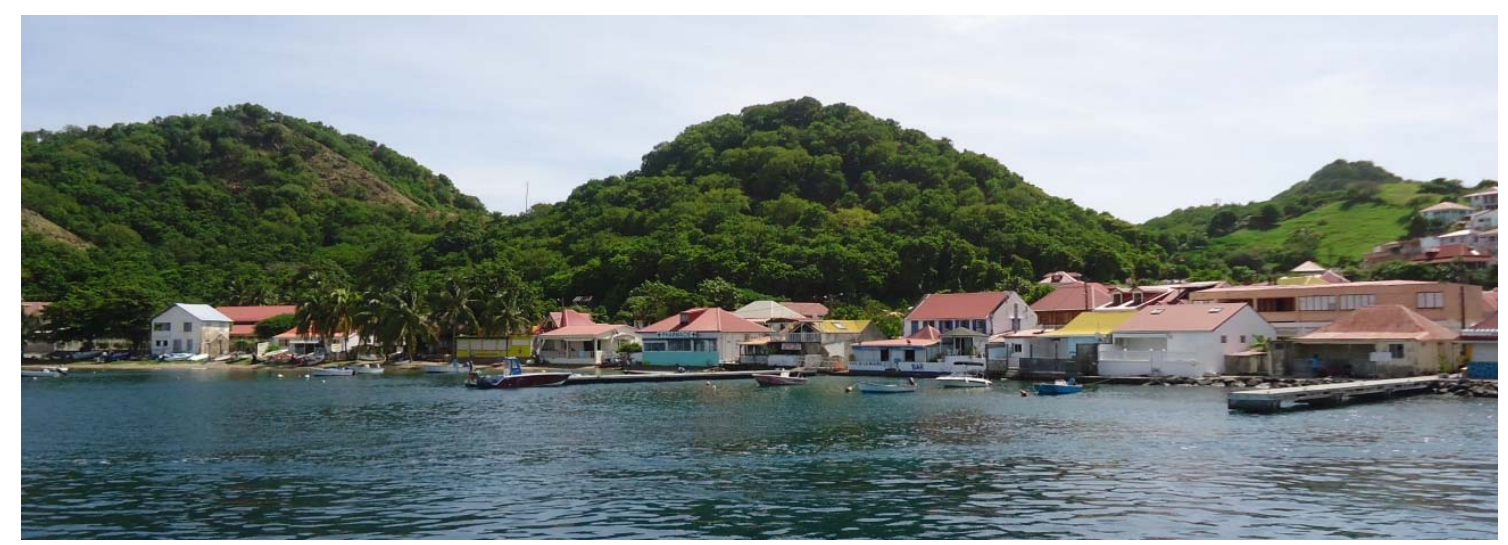

Figure 2. Antilles : Habitat sur la plage "les pieds dans l'eau", (cliché $^{\odot}$ J.-M. Beynet, 2013).

\subsection{Que ce soit en Polynésie ou aux Antilles}

Si le littoral n'est pas protégé par un lagon, il est rocheux le plus souvent et généralement, les infrastructures routières et habitats ne sont pas calés trop près, ni trop bas sur la mer, justement pour tenir compte des marées de tempête. Si le littoral est protégé par une barrière récifale formant lagon, le récif est souvent affleurant et les algues qui forment la crête algale, les corallines, sont capables de s'adapter doucement à une lente montée des 


\section{Thème 7 - Risques côtiers}

eaux en se développant et en s'élevant, elles-aussi, au fur et à mesure que l'eau monte lentement. De ce fait, plusieurs décennies plus tard, même avec une montée du niveau de la mer de quelques dizaines de centimètres, le niveau de protection contre la houle est sensiblement le même et c'est toujours la submersion par les marées de tempête qui impacterait le plus le littoral bâti. A condition toutefois, que les activités humaines et la dégradation de la qualité de l'eau, n'altèrent pas ces mécanismes naturels. Il est important de noter par ailleurs l'effet bénéfique des tempêtes dans certains cas, en particulier dans les zones classées en réserves naturelles. A Saint-Martin par exemple, lors du passage d'Irma fin 2017, les zones protégées ont été beaucoup moins endommagées par la houle cyclonique que certaines zones habitées. Les scientifiques le reconnaissent : "Partout où c'est possible, il faut redonner de la place aux écosystèmes, par la relocalisation du bâti, afin de reconstituer une bande côtière non perturbée par l'homme et ayant, en autres fonction, celle d'amortir les effets des tempêtes" (DUVAT, 2018). Selon Météo France, l'incidence des tempêtes est positif en terme d'apports de matériaux (notamment coralliens) sur les littoraux "naturels", où les blocailles s'épandent et participent à l'engraissement. Au contraire, des protections souvent mal conçues, conduisent à des affouillements et empêchent les dépôts de blocailles.

\section{Urbanisme réglementaire pour les infrastructures et les habitats}

\subsection{Aux Antilles françaises}

Dans nos départements d'outre-mer, dès le XVII ${ }^{\text {ème }}$ siècle, le littoral a été protégé par la règle des cinquante pas géométriques, qui prévaut encore de nos jours. Aujourd'hui, si le statut foncier de ces espaces est déterminé par les dispositions figurant dans la $5^{\text {ème }}$ partie du code général de la propriété des personnes publiques (CG3P), le code de l'urbanisme leur réserve des dispositions particulières.

Ainsi l'article L. 5111-1 du CG3P indique que "La zone comprise entre la limite du rivage de la mer et la limite supérieure de la zone dite des cinquante pas géométriques définie à l'article L. 5111-2 fait partie du domaine public maritime de l'Etat".

L'article L. 5111-2 définit les limites de la "réserve domaniale dite des cinquante pas géométriques" espace spécifique qui "est constitué par une bande de terrain délimitée dans les départements de La Réunion, de la Guadeloupe et de la Martinique. Elle présente dans le département de la Guyane une largeur de 81,20 mètres comptée à partir de la limite du rivage de la mer tel qu'il a été délimité en application de la législation et de la réglementation en vigueur à la date de cette délimitation".

Mais c'est l'article L121-45 du code de l'urbanisme qui apporte les précisions nécessaires: "Il est déterminé une bande littorale comprise entre le rivage de la mer et la limite supérieure de la réserve domaniale dite des cinquante pas géométriques" définie à l'article L. 5111-2 du code général de la propriété des personnes publiques et, à Mayotte, à l'article L. 5331-4 de ce code. A défaut de délimitation ou 


\section{XVI'èmes Journées Nationales Génie Côtier - Génie Civil \\ Le Havre 2020}

lorsque la réserve domaniale n'a pas été instituée, cette bande présente une largeur de 81,20 mètres à compter de la limite haute du rivage. Les terrains situés dans les parties urbanisées de la commune comprises dans la bande littorale définie à l'article L. 12145 sont préservés lorsqu'ils sont à l'usage de plages, d'espaces boisés, de parcs ou de jardins publics. Il en est de même des espaces restés naturels situés dans les parties urbanisées de la bande littorale, sauf si un intérêt public exposé au plan local d'urbanisme justifie une autre affectation.

D'autres dispositions du CG3P préservent les droits des tiers et ne n'appliquent pas à des biens appartenant à des personnes publiques ou privées pouvant justifier de leur droit (art. L5111-3 et L5111-4).

Le code de l'urbanisme permet dans les 50 pas et dans des zones délimitées par le PLU l'affectation d'espaces "à des services publics, des équipements collectifs, des opérations de réaménagement de quartier, de logement à caractère social et de résorption de l'habitat insalubre, des commerces, des structures artisanales, des équipements touristiques et hôteliers ainsi qu'à toute autre activité économique dont la localisation à proximité de la mer est justifiée par son usage ou par une nécessité économique de desserte par voie maritime. Dans ce cas, des mesures compensatoires permettant le maintien de l'équilibre du milieu marin et terrestre sont mises en œuvre. Ces installations organisent ou préservent l'accès et la libre circulation le long du rivage". Dans ces secteurs, sont autorisés l'adaptation, le changement de destination, la réfection, la reconstruction et l'extension limitée des constructions existantes.

(art. L121-48 c. urb.).

Les adaptations sont également possibles dans les secteurs occupés par une urbanisation diffuse, à condition qu'ils soient identifiés dans la partie du schéma régional valant SMVM (Schéma de Mise en Valeur de la Mer). Ces secteurs doivent "(...) être affectés à des services publics, des équipements collectifs, des programmes de logements à caractère social, des commerces, des structures artisanales, des équipements touristiques et hôteliers. Dans ce cas, des mesures compensatoires permettant le maintien de l'équilibre du milieu marin et terrestre sont mises en ouvre. Ces installations organisent ou préservent l'accès et la libre circulation le long du rivage. Dans ces secteurs, sont autorisés l'adaptation, le changement de destination, la réfection, la reconstruction et l'extension limitée des constructions existantes", (art. L121-49 c. urb.).

En Guadeloupe et Martinique, l'Agence des Cinquante Pas géométrique assure la gestion de cette zone. En 2016, une mission conjointe du CGAAER (Conseil Général de l'Alimentation, de l'Agriculture et des Espaces Ruraux) et du CGEDD (Conseil Général de l'Environnement et du Développement Durable) a été chargée d'évaluer la préservation de la biodiversité et la maîtrise foncière sur cette bande littorale. Ce rapport précise que le cas de Saint-Martin, devenue collectivité d'outre-mer, montre que l'affectation de l'ensemble des pas géométriques au Conservatoire du Littoral permet une 


\section{Thème 7 - Risques côtiers}

protection forte et durable. Cependant les dégâts causés par le cyclone Irma en septembre 2017, montrent que le problème n'est pas simple et que la modification du PPRN (Plan de Prévention contre les Risques Naturels), n'est pas acceptée ni par les populations, ni par la Collectivité de Saint-Martin. A "Sandy-Ground", de l'habitat en dur avait été construit en bordure du littoral au plus près des plages. La plupart des maisons ont été détruites par le déferlement de la houle cyclonique. Elles sont en ruine à présent. Et malgré cela, les riverains refusent de partir et veulent reconstruire au même endroit. L'ancien PPRN datait de 2011. Après le passage du cyclone "Irma", il a été révisé pour mieux prendre en compte le risque de submersion marine. Ce nouveau PPRN a redéfini les zones à risques et surtout, celles en bord de mer où toute reconstruction sera interdite, ce que refusent la population et la Collectivité de Saint-Martin. En métropole, dans un rapport récent (BUCHOU, 2019), des propositions ont été faites au Gouvernement pour "repenser l'élaboration de nos politiques publiques sur le littoral, face à l'érosion côtière". Ce rapport préconise plusieurs recommandations en tenant compte de la dynamique littorale et du Code de l'Urbanisme, pour assurer par étapes, la recomposition spatiale des territoires impactés par l'érosion côtière. Cela ne sera pas facile à mettre en œuvre, car des riverains devront quitter le littoral et reculer, en abandonnant non seulement leurs biens immobiliers, mais également, leur travail, leur culture et leurs racines pour certains. Dès 2019, des tentatives de retrait stratégique d'habitations trop proches de la mer sont vécues douloureusement par les riverains, tant en France métropolitaine qu'outremer.

\subsection{En Polynésie française}

En Polynésie, la réglementation se base essentiellement, pour ne pas dire uniquement, sur les Plans de Prévention des Risques. Établis par le Pays, ils doivent être validés par les Communes. Ces derniers comportent trois volets : les risques d'instabilité, à terre, devant anticiper les éboulements ou chutes de blocs, les risques d'inondations de rivières et enfin les risques de submersion océanique, liés à la houle.

Ces plans ont été établis sur la base d'enquêtes historiques, recensant par Communes l'ensemble des atteintes aux personnes et aux biens de mémoire d'hommes. Ils ont été complétés par un travail de cartographie, en reconnaissance de terrain pour les instabilités ou d'après modélisation $1 \mathrm{D}$ ou $2 \mathrm{D}$ pour les rivières. En termes de submersion océanique, le travail réalisé est assez sujet à caution, la méthodologie reste floue et a conduit dans l'archipel des Tuamotu par exemple, à classer en risque maximum une bande de $30 \mathrm{~m}$ de large coté océan et $10 \mathrm{~m}$ coté lagon, sans tenir aucun compte des protections naturelles comme la levée détritique ou la protection que peut "offrir" un atoll à un autre en étant situé à l'amont des champs de houles. Une seule commune a validé d'ailleurs son Plan de Prévention des Risques ! L'absence d'une véritable instruction, d'une interprétation très rigoureuse des textes mais également la déresponsabilisation des acteurs communaux (seule la Commune de Papeete peut signer des permis de construire), ont conduit à une 


\section{XVI'̀mes Journées Nationales Génie Côtier - Génie Civil \\ Le Havre 2020}

absence de régulation. Avec la pression foncière et notamment l'indivision, le nombre de constructions exposées, d'habitation ou à vocation touristique (pension) augmentent considérablement. Il est d'ailleurs intéressant de faire un lien avec l'époque de la perliculture où de nombreux bâtis ont été réalisés sur des pinacles ou en bordure de lagon. L'utilisation des photographies aériennes est à ce sens très éloquente. Il n'existe pas de cadre ou de schéma préservant ou régulant le littoral, et d'ailleurs, le SAGE est en cours de rédaction. Il est difficile de parler de cadre réglementaire. Les Communes disposent d'un outil, le PGA (Plan Général d'Aménagement, semblable aux anciens POS métropolitains), mais sa mise en œuvre est loin d'être généralisée. Toutes les communes de Tahiti n'en disposent pas! Il est par exemple inexistant à Bora Bora, moteur du tourisme polynésien. Soulignons enfin qu'en France métropolitaine, l'habitat sur pilotis sur le Domaine Public Maritime n'est pas autorisé. Tel n'est pas le cas en Polynésie française où il existe un contexte réglementaire spécifique pour le droit des lagons (CAZALET, 2008).

\section{Conclusions}

Aux Antilles, comme en Polynésie, l'habitat précaire, trop près des plages (et même sur les plages parfois comme à Sandy-Ground à Saint-Martin) est très vulnérable vis-à-vis des risques de submersion marine. En revanche, il semblerait que l'habitat résidentiel, soit moins exposé, car il est généralement calé plus haut que certains sites de métropole où des marinas créées dans les années 70 ont été construites en dur, très basses sur l'eau en régions PACA et Occitanie. De plus, la culture du risque de submersion marine par les populations des Antilles et de Polynésie est certainement plus développée depuis plusieurs siècles qu'en métropole où les populations découvrent ce type de problèmes dans des zones identifiées comme vulnérables depuis peu. L'image de l'habitat en bord de mer, sur pilotis ou non, est mis à mal par la crainte de la submersion. La montée des eaux est très souvent perçue comme l'une des principales menaces du changement climatique. Pourtant, ce sont aujourd'hui les précipitations diluviennes, les vents et les instabilités de terrain qui causent le plus de pertes humaines et génèrent le plus de dégâts. Le littoral est un lieu emblématique, d'images de plages et de nature. Mais l'occupation humaine n'est pas forcément dégradante. Bien pensée, elle pourrait pourtant être une solution dans des sites adéquats et un laboratoire d'innovations et de développement. Les enjeux liés à la montée des eaux, indéniable et bien plus rapide qu'aucun des épisodes précédents dans les temps géologiques, doivent conduire à innover et repenser notre occupation du littoral. Le développement des infrastructures sur pilotis est bien moins impactant qu'une reprise de littoral, avec digues et protections lourdes, qui sont consommatrices de ressources et conduisent souvent à des impacts directs ou indirects bien plus importants. Enfin, il parait important de souligner que les îles de Polynésie et des Antilles sont très dépendantes de leurs ports et aéroports. Ces grandes infrastructures de transport sont vitales pour ces îles isolées de la métropole. Et malheureusement les 


\section{Thème 7 - Risques côtiers}

pistes des aéroports sont souvent très basses par rapport au niveau de la mer. Certaines d'entre-elles devront probablement être surélevées à court ou moyen terme.

\section{Références bibliographiques}

BUCHOU S. (2019). Quel littoral pour demain? Vers un nouvel aménagement des territoires côtiers adapté au changement climatique, Rapport du Député M. S. BUCHOU remis à M. Le Premier Ministre et à Madame la Ministre de la Transition Ecologique et Solidaire, Octobre 2019, 113 p.

CAZALET B. (2008). Droit des lagons de Polynésie française, Revue juridique de l'Environnement, Année 2008, volume 4, pp 391-407. https://doi.org/10.3406/rjenv.2008.4777

DUVAT V. (2018). Etudier les impacts des cyclones pour mieux appréhender les trajectoires de vulnérabilité et d'adaptation des îles tropicales au changement climatique, Journée thématique "Cyclones et changement climatique", Labex L-IPSL Paris, Jussieu, février 2018, 31p. cse.ipsl.fr > files > journee_cyclones > Cyclones_6fev2018_Duvat LABROSSE S. (2010). Des îles hautes aux atolls : une histoire du plancher océanique. https://planet-terre.ens-lyon.fr/article/ile-haute-atoll.xml OPPENHEIMER M., GLAVOVIC B.C., HINKEL J., VAN DE WAL R., MAGNAN A.K., ABD-ELGAWAD A., CAI R., CIFUENTES-JARA M., DE CONTO R.M., GHOSH T., HAY J., ISLA F., MARZEION B., MEYSSIGNAC B., SEBESVARI Z., GIEC (2019). Sea level rise and implications for low lying islands, Coasts and Communities, In: IPCC Special Report on the Ocean and Cryosphere in a Changing Climate, Chapter 4, 51 ${ }^{\text {st }}$ Session held on 20-28 September 2019, pp 321-445.

PAGNEY BENITO-ESPINAL F. (2019). Construire une culture du risque efficiente? Le cas de la Guadeloupe et de la Martinique, Géo confluences, décembre 2019, 8p. http://geoconfluences.ens-lyon.fr/informations-scientifiques/dossiers-thematiques/risques-et-societes/articlesscientifiques/culture-du-risque-antilles 